\title{
An dissipated energy based analysis of fatigue crack propagation law
}

\author{
Aleksei Vshivkov ${ }^{11}$, Anastasiia Iziumova ${ }^{1}$, Rustam Yarullin ${ }^{2}$, Valery Shlyannikov ${ }^{2}$, Oleg \\ Plekhov ${ }^{I}$ \\ ${ }^{1}$ Institute of Continuous Media Mechanics of the Ural Branch of Russian Academy of Science, \\ 614013 Perm Academika Koroleva st, 1, Russia \\ ${ }^{2}$ Institute of Power Engineering and Advanced Technologies, FRC Kazan Scientific Center, Russian \\ Academy of Sciences, 420111 Kazan ul. Lobachevskogo, 2/31, POB 261, Russia
}

\begin{abstract}
The experimental study of heat flux evolution at the fatigue crack tip during biaxial loading was carry out in this work. The plane samples of stainless steel AISI 304 with thick of $3 \mathrm{~mm}$ were weakened by notch to initiate fatigue crack at the centre of samples. A contact heat flux sensor based on the Seebeck effect was used to monitor the dissipated thermal energy. During tests the samples were subjected to cyclic loading of $5 \mathrm{~Hz}$ with constant stress amplitude and different biaxial parameter. The experimental results confirm the previous conclusions of authors about two regime of energy dissipation at fatigue crack tip. The curve of the dissipated energy can be divided in two stages. In the second stage is characterized by classical linear relation between crack rate and energy dissipation. In the first stage the crack rate is proportional to the multiplication of the power of heat flux by crack length. The energy dissipation does not depend on the biaxial parameter during cyclic loading.
\end{abstract}

\section{Introduction}

Technical progress, complication of mechanisms and designs, ambitious projects in the field of mechanical engineering, aircraft construction, nuclear energy, and space exploration contributed to the active development of many fields of science and technology, including the fracture mechanics. A number of approaches has been developed to study the processes of nucleation and propagation of fatigue cracks [1-4].

It is well known that real metals have a complex structure, which is a hierarchy of different scale levels. Under deformation, the structural evolution is observed at all scale levels and leads to irreversible deformation and failure that is accompanied by energy accumulation and dissipation. Investigation of thermodynamics of deformation and failure is a key issue in solid mechanics. The analysis of the kinetics of damage accumulation, the process of crack nucleation and kinetics of the crack development allows specialists to predict the time of structure failure and to perform in proper time a partial replacement or repair of deteriorated units of complex structures. Moreover, the repair or replacement of 
the worn-out parts on a timely basis is more effective than their complete replacement after mechanical damage. It is therefore very important to know the time during which the defects in the ill-behaved areas are reaching critical values.

Criteria of fracture mechanics are generally based on elastic and elasto-plastic solutions for simplified geometries and idealized loading conditions. There criteria allows one to predict the peculiarities of the processes of initiation and propagation of fatigue cracks [5-8] both in uniaxial and complex types of loading, combining mode 1 and 2, [1,9]. It gives a good result in many engineering application but don't describe in detail the physics of fatigue and fracture. As a result, it requests the application of safety coefficients in engineering practice.

So, it is of considerable interest to study the behavior of materials under mixed loading conditions that combine mode 1 and 2 . In sufficiently plastic structural materials the propagation of the crack begins when the plastic deformation near its tip becomes large (of the order of a 10 percent). This irreversible process is accompanied by the release and accumulation of energy, which leads to a local temperature change in the region of the crack tip and the appearance of a heat flux.

For a long time, infrared thermography is regarded as the most effective method for estimating the power of the heat sources in the process of mechanical testing $[9,10]$. The principal solution of the problem of energy dissipation measurement under deformation and failure can be reach by the development of additional system for direct monitor of heat flow [11]. The heat generation process depends on both the thermo elastic effect and plastic energy dissipation. The measurement of heat flux near the crack tip allows one to calculate the energy balance under crack propagation and to propose a new equation for crack propagation. Attempts to propose the new equation for crack propagation were made by many authors. They used such quantities as the J-integral, the work of plastic deformation, the size of the zone of plastic deformation, the amount of dissipated energy and other [1214]. The classical assumption of an almost complete dissipation of the energy of deformation into heat [15] turns out to be correct only in a limited number of cases.

The previous authors' investigations were focused on crack growth problems under an opening or mode I mechanism [16] and a relationship was proposed for the growth rate of a fatigue crack based on an analysis of the energy balance at its tip. However, most structures are failed due to mixed mode loading. Many uniaxial loaded materials, structures and components often contain randomly oriented defects and cracks which develop a mixed mode state by rotation of their orientation with respect to the loading axis.

This work is devoted to the investigation of the dissipated energy in the process of crack propagation under biaxial loading. For this purpose, the original contact heat flux sensor was adopted to detect energy dissipation value in the process of crack propagation under biaxial testing machine and verify the data of infrared thermography.

The theoretical study of stress field at crack tip allows us to divide the plastic work and, as a consequence, energy dissipation at crack tip into two parts corresponding to reversible (cyclic) and monotonic plastic deformation. Analysis of these processes has shown the independence of energy dissipation in cyclic plastic zone from the crack growth. Dissipation in the monotonic plastic zone is a linear function of crack rate. The proposed analysis is based the hypothesis about link between the elastic solution and the elasticplastic deformation in the fatigue crack tip using secant plasticity modulus. As a result, it gives a way to correlate energy dissipation and fatigue crack rate under both one dimension and multidimensional loading.

Analysis of the experimental data on crack propagation under biaxial loading reveals the similar qualitative features of energy dissipation under uniaxial and biaxial loading conditions. We observed two stages of energy dissipation: constant value at the first stage 
and sharp increase at its final stage. The comparison of the phenomenological approximation shows good qualitative agreement under all studied loading conditions.

\section{Experimental setup}

A series of cruciform samples made from stainless steel AISI 304 were tested. During tests the samples were subjected to cyclic loading of $5 \mathrm{~Hz}$ with constant stress amplitude and different biaxial coefficient $\eta=P x / P y(1,0.5,0)$ and ratio $\mathrm{R}=0.1$. The crack length in the course of the experiment was measured by optic method.

To analyze the dissipated energy at the crack tip a contact heat flux sensor was designed and assembled. The sensor is based on the Seebeck effect, which is the reverse of the Peltier effect.

The Peltier effect is a thermoelectric phenomenon, in which the passage of electric current through conducting medium leads to the generation or absorption of heat at the point of contact (junction) of two dissimilar conductors. The quantity of heat and its sign depend on the type of materials in contact, the direction and the strength of the electric current.

The quantity of heat absorbed or dissipated by the element is directly proportional to the current intensity and the time of its passage:

$$
P=\Pi_{A B} I
$$

where $\mathrm{P}$ - the power of heat flux, I - the direct current, $\Pi_{A B}$ - Peltier coefficient.

This sensor were calibrated using a device with a controlled heat flux. A wire resistor with the known resistance is glued on a plastic plate with a size equal to that of test samples. The heat isolating system provides the heat flux from the resistance to the sensor only. The heat flow was calculated using the values of the resistor voltage and the electric current across the resistor.

\section{Results}

During the experiments, a series of samples was tested. The table 1 shows loading conditions of experiments. The crack length and the heat flux were recorded. The crack rate was $10-7-10-4 \mathrm{~m} /$ cycle during the crack propagation.

Table 1. Setting Word's margins.

\begin{tabular}{|c|c|c|c|c|}
\hline № & Load, $\mathbf{k N}$ & Biaxial parameter & Cycles & Crack length, $\mathbf{m m}$ \\
\hline 1 & 47 & 0 & 120000 & 45.2 \\
\hline 3 & 47 & 1 & 259000 & 41.7 \\
\hline 4 & 47 & 0,5 & 155000 & 43.6 \\
\hline 5 & 47 & 0.5 & 190000 & 45.9 \\
\hline
\end{tabular}


A dissipated energy during the tests is presented in figure 1a. There is two characteristic stages (solid and dotted lines) corresponding different character of heat dissipation. Circle markers show the point of changes of energy dissipation stages. On the Paris curve, the point of change of the stages is clearly not pronounced, figure $2 b$. The Energy dissipation change point is determined by the maximum value of the curvature of the energy dissipation curve.

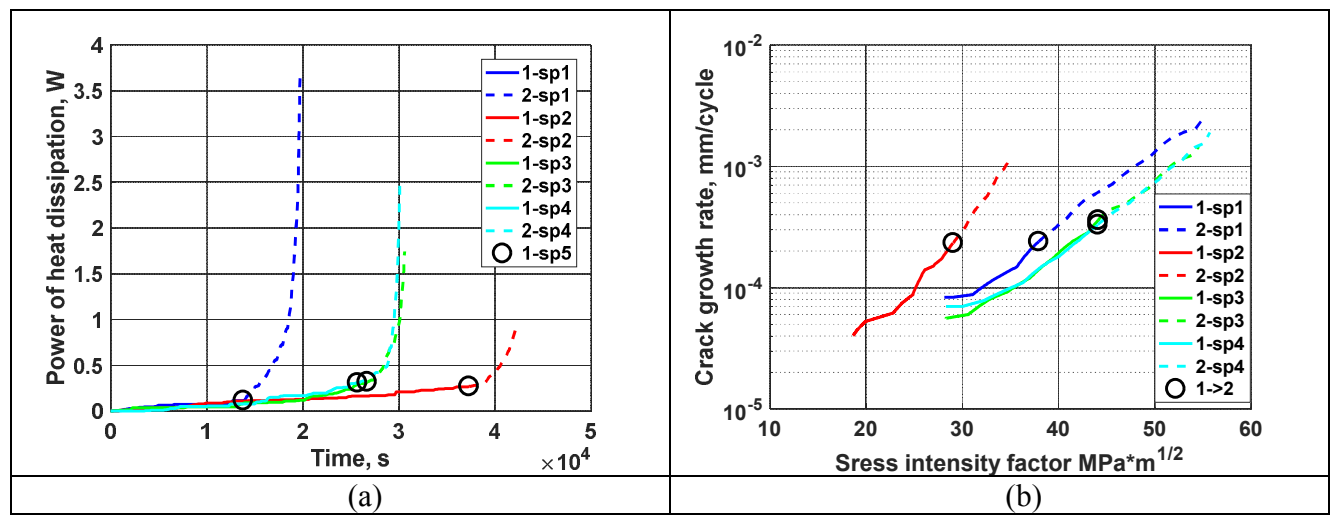

Fig. 1. Results of fatigue test: dissipated energy(a) and Paris curve (b).

After analysing the experimental data, based on the hypothesis about the stages of fatigue crack propagation [11], characteristic relationships between the crack growth rate and heat dissipation in both stages were observed. At the first stage, the crack rate is proportional to the multiplication of the dissipated energy and crack length (figure 2a), in the second stage there is linear relation between dissipated energy and crack growth rate (figure 2b).

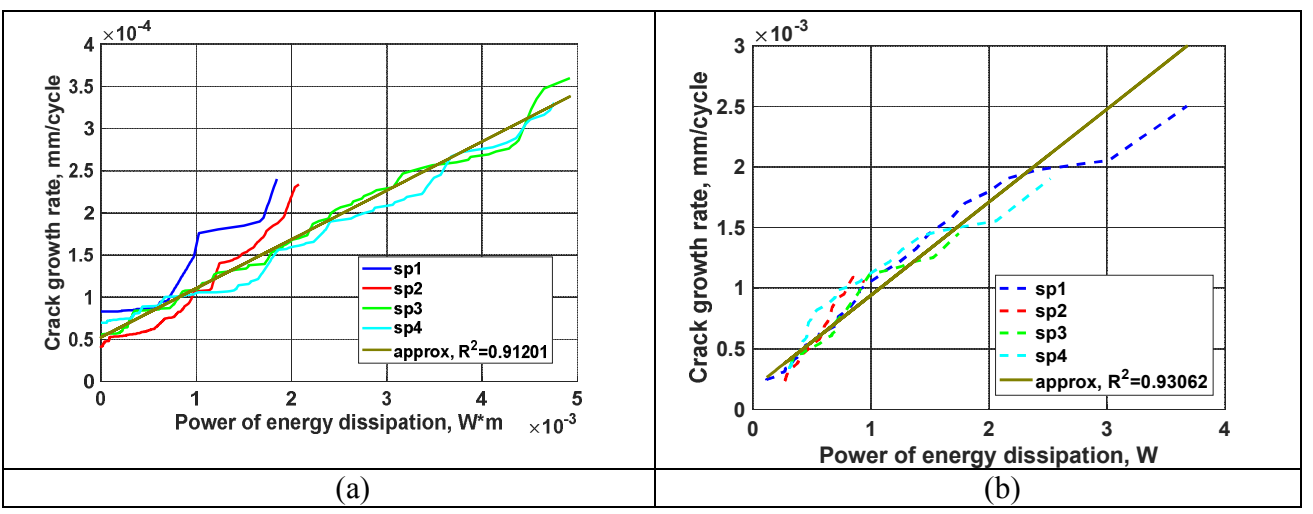

Fig. 2. Relation between crack rate and heat dissipation on first (a) and second (b) stages.

\section{Approximation of energy dissipation at crack tip under arbitrary loading conditions}

Following the [17], we can propose a link between elastic and full strain at the crack tip as 
$\varepsilon_{i j}^{e f}=\left(\frac{G}{G_{s}}\right)^{\frac{1}{2}} \varepsilon_{i j}^{e l}$

where $G$ - the shear modulus, $G_{S}$ - secant shear modulus, $\varepsilon_{i j}^{e l}$ - elastic strain tensor, $\varepsilon_{i j}^{e l}$ experimentally calculated strain measure.

Using the Ramberg-Osgood relationship $\gamma=\frac{\tau}{G}+A\left(\frac{\tau}{\tau_{0}}\right)^{n}$, we can write the following estimation for octahedral stress and link it with an elastic solution

$\tau_{o c t}=\frac{\left(1+\frac{2(1+v)}{3} B \xi^{n-1}\right)^{\frac{1}{2}}}{1+B \xi^{n-1}} \tau_{o c t}^{e l}$,

where $A, \tau_{0}, n$ - material constants, $B=\frac{G A}{\tau_{0}}\left(\frac{\tau_{e}}{\tau_{0}}\right)^{n}, \xi=\frac{\tau_{o c t}}{\tau_{e}}, \tau_{e}$ - elastic limit.

Under cyclic loading, we have to divide energy dissipation in cyclic and monotonic plastic zones at the crack tip

$U_{p}^{t o t}=U_{p}^{c y c}+U_{p}^{m o n}$

The energy of representative volume at cyclic zone can be estimated as

$U_{p}^{c y c}=\frac{3}{2} \tau_{e c} \gamma_{p c}$

where $\tau_{e c}$ - characteristic size of the yield surface, $\gamma_{p c}=\left(\tau_{o c t, c}-\tau_{e c}\right)\left(\frac{1}{G_{S}}-\frac{1}{G}\right)-$ amplitude of plastic deformation under an assumption of the validity of Ramberg-Osgood relationship ship, $\tau_{o c t, c}$ - stress change in the representative volume.

The energy of cyclic plastic zone can be calculated as a double integral over the region (S) bounded on the outside of the monotonic plastic deformation zone and inside of the fracture zone

$U_{p}^{c y c}=2 \int_{S} \int_{0}^{\pi} \frac{3}{2} \tau_{e c}^{2}\left(\frac{1}{G_{s}}-\frac{1}{G}\right)\left(\frac{\tau_{o c t, c}}{\tau_{e c}}-1\right) r d r d \theta$

Using definition $\alpha=\frac{\tau_{o c t}^{e l}}{\tau_{e}}=\frac{K f_{e}^{\frac{1}{2}}}{3 \sqrt{r} \tau_{e}}=\left(\frac{r_{p} f_{e}}{r}\right)^{\frac{1}{2}}$ (here $K-$ stress intensity factor, $r_{p}$ estimation for plastic zone size, $r, \theta$ - polar coordinates, $f_{e}$ - function of polar coordinate $\theta$, 
we can approximate of plastic zone boundary as $r=r_{p, c} f_{e}$, for cyclic-fracture zone boundary $r=r_{p, c} f_{e} \frac{\tau_{e c}}{\tau_{f r}}$.

The energy increment in cyclic plastic zone can be written as

$$
\frac{d U_{p}^{c y c}}{d N}=3 \tau_{e c}^{2}\left(\frac{1}{G_{S}}-\frac{1}{G}\right) \int_{S} \int_{0}^{\pi} \frac{d \tau_{o c t, c}}{d \tau_{o c t}^{e l}} \frac{d \tau_{o c t}^{e l}}{d N} r d r d \theta
$$

The integration of equation (7) gives $\frac{d U_{p}^{c y c}}{d N}=0$. It means that dissipation in cyclic plastic zone doesn't depend on the crack advance but it is fully determined by the applied load. The energy dissipation in monotonic plastic zone can be estimated as

$U_{p}^{m o n}=\frac{3}{2} \tau_{e} \gamma_{p}$

Energy increment per one cycle can be written as

$$
\frac{d U_{p}^{m o n}}{d N}=3 \tau_{e}\left(\frac{1}{G_{S}}-\frac{1}{G}\right) \int_{S_{1}}^{\pi} \int_{o c t}^{\pi} \frac{d \tau_{o c t}}{d \tau_{o c t}^{e l}} \frac{d \tau_{o c t}^{e l}}{d l} \frac{d l}{d N} r d r d \theta
$$

Integration of equation (9) for the case of $\frac{d r_{p}}{d a} \rightarrow 0$ gives linear relationship between energy increment and crack rate. Finally, the equations (8) and (9) allow us to propose the following approximation for energy of plastic deformation at the fatigue crack tip

$$
\frac{d U_{p}^{\text {tot }}}{d N}=W_{1}\left(A_{\tau}^{2}, r_{p}\right)+W_{2}\left(A_{\tau}^{2}, r_{p}\right) \frac{d l}{d N}=a_{1} A_{\tau}^{2}+a_{2} A_{\tau}^{2} \frac{d l}{d N}
$$

where $A_{\tau}$ - applied stress amplitude which determines the diameter of yield surface, $a_{1}, a_{2}-$ material constants.

The equations (8) and (9) are written based on the classical elastic solution for crack tip. As a results, they could be integrated for arbitrary loading conditions and allows one to estimate the energy dissipation as a function of crack tip under both uniaxial and multiaxial loading. We have experimentally proved this proposition. Figures 3 presents the comparison of approximation (10) and results of the experimental study. There is the power of heat flux during fatigue crack propagation under biaxial loading (experimental measurement and theoretical calculation) with different biaxial parameter $\mathrm{n}=0,0.5,1$. 


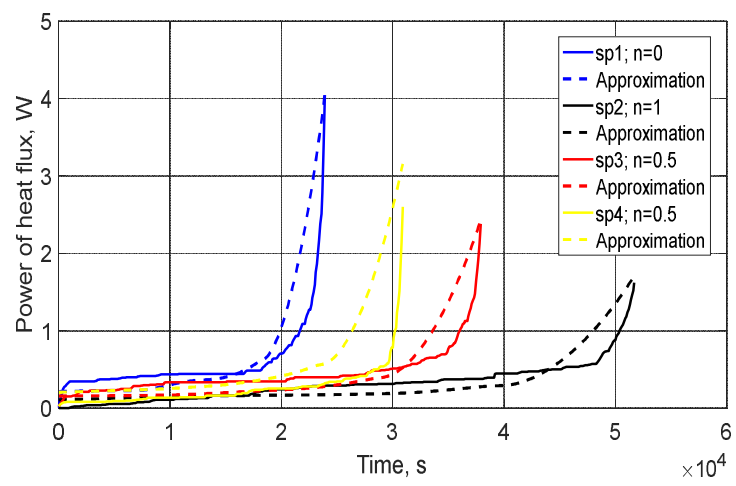

Fig. 3. Energy dissipation histories during fatigue test of steel samples (Solid line - experimental results, dotted line-approximation (10)).

The results confirm the hypothesis of the existence of two stages of energy dissipation during the fatigue cracks propagation under both uniaxial and multiaxial loading conditions. The first stage corresponds to slow cracks (about $1 \mathrm{e}-7 \mathrm{~m} /$ cycle) and there is linear relation between crack growth rate and multiplication of energy dissipation and crack length. The second stage corresponds to crack rate more then $1 \mathrm{e}-6 \mathrm{~m} /$ cycle and it is characterised by classical linear relation between crack growth rate and energy dissipation.

\section{Conclusion}

A series of experiments was carried out to study the dissipation of energy at the fatigue crack tip under biaxial loading. The experimental technique, based on a contact heat flux sensor was adopted for measuring the energy dissipation during fatigue test under biaxial loading. The results confirm the hypothesis of the existence of two stages of energy dissipation during the fatigue cracks propagation. The first stage corresponds to slow cracks (about 1e-7 $\mathrm{m} /$ cycle) and there is linear relation between crack growth rate and multiplication of energy dissipation and crack length. The second stage corresponds to crack rate more then $1 \mathrm{e}-6 \mathrm{~m} /$ cycle and it is characterised by classical linear relation between crack growth rate and energy dissipation. Based on the experimental results, it is possible to generalize the hypothesis about linear relation between energy dissipation at the fatigue crack tip and crack rate to the case of biaxial loading.

To explain the generalization of the hypothesis we propose a theoretical description of energy dissipation at crack tip based on the link between experimentally observed strain field and corresponding elastic solution. It give as a possibility to introduce the approximation of energy dissipation at crack tip as a function of crack rate under arbitrary loading conditions. The experimental results confirm the theoretical consideration and exhibit linear relation between energy dissipation at the fatigue crack tip and crack rate under all investigated loading conditions (biaxial coefficients).

\section{Acknowledgments}

This work was supported by the grant of the Russian Foundation for Basic Research [grant number 16-51-48003]. 


\section{References}

1. J.R. Yates, M. Zanganeh, R.A. Tomlinson, M.W. Brown, F.A. DiazGarrido, Crack paths under mixed mode loading, Engineering Fracture Mechanics, 75, 3-4, 319-330 (2008).

2. M. Mokhtarishirazabad, P. Lopez-Crespo, B. Moreno, A. Lopez-Moreno, M. Zanganeh, Optical and analytical investigation of overloads in biaxial fatigue cracks, International Journal of Fatigue, 100, 2, 583-590 (2017).

3. Y. Izumi, T. Sakagami, K. Yasumura, D. Shiozawa, A new approach for evaluating stress intensity factor based on thermoelastic stress analysis, APCFS/SIF, 47-51 (2014).

4. J.S. Short, D.W. Hoeppner, A Global/local theory of fatigue crack propagation, Engineering Fracture mechanics, 33, 2, 175-184 (1989).

5. P.C. Paris, M.P. Gomez, W.E. Anderson, A rational analytic theory of fatigue, The Trend in Engineering, 13, 9-14 (1961).

6. A. Chudnovsky, A. Moet, Thermodynamics of translational crack layer propagation, J. Materials Science, 20, 630-635 (1985).

7. Yu.G. Matvienko, E.M. Morozov, Calculation of the energy J-integral for bodies with notches and cracks, International Journal of Fracture, 125, 249-261 (2004).

8. V. Shlyannikov, A. Tumanov, A. Zakharov, A. Gerasimenko, Surface flaws behavior under tension, bending and biaxial cyclic loading, International Journal of Fatigue, 92, 2, 557-576 (2016).

9. G. Meneghetti, M. Ricotta, Evaluating the heat energy dissipated in a small volume surrounding the tip of a fatigue crack, International Journal of Fatigue, 92, 2, 605-615 (2016).

10. A. Risitano, G. Risitano, Cumulative damage evaluation in multiple cycle fatigue tests taking into account energy parameters, International Journal of Fatigue, 48, 214-222 (2013).

11. C. Pradere, M. Joanicot, J-C. Batsale, J. Toutain, C. Gourdon, Processing of temperature field in chemical microreactors with infrared thermography, QIRT Journal, 3, 117-135 (2006).

12. P. Rosakis, A.J. Rosakis, G. Ravichandran, J. Hodowany, A thermodynamic internal variable model for the partitional of plastic work into heat and stored energy in metals, J. Mech. Phys. Solids, 48, 581-607 (2000).

13. W. Oliferuk, M. Maj, B. Raniecki, Experimental Analysis of Energy Storage Rate Components during Tensile Deformation of Polycrystals, Materials Science and Engineering, 374, 77-81, (2004).

14. A. Izyumova, O. Plekhov, Calculation of the energy J-integral in plastic zone ahead of a crack tip by infrared scanning, FFEMS, 37, 1330-1337 (2014).

15. W.S. Farren, G.I. Taylor, The heat developed during plastic extension of metals, Proc. Royal. Soc. of London. Ser., 107, 422-451 (1925).

16. A. Vshivkov, A. Iziumova, U. Bar, O. Plekhov, Experimental study of heat dissipation at the crack tip during fatigue crack propagation, Fracture and Structural Integrity, 35, 131-137 (2016).

17. K.N. Raju, An energy balance criterion for crack growth under fatigue loading from considerations of energy of plastic deformation, International Journal of Fracture Mechanics, 8(1), 1-14 (1972). 